\title{
Indicadores de calidad en cáncer de mama*
}

\author{
Drs. ANA MARÍA DONOSO R. ${ }^{1}$, MARCELA AMAR C. ${ }^{1}$, MATÍAS MINASSIAN M. ${ }^{1,2}$, \\ Al. CLAUDIO SALAZAR M. ${ }^{3}$, Drs. DOUGLAS ARBULO L. ${ }^{1,2}$, JEANNIE SLATER M. ${ }^{4}$, \\ RICARDO SCHWARTZ J. ${ }^{1}$, LIONEL GÓMEZ S. ${ }^{1}$, KUEN LEE C. ${ }^{1}$
}

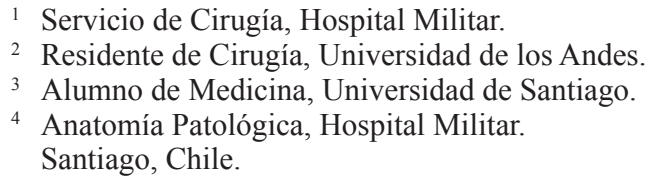

Abstract

\section{Quality indicators in breast cancer surgery}

Introduction: Although clear guidelines for breast cancer management have been developed and widespread, there are many variations between centers and even among breast cancer surgeons, with impact in clinical outcomes. Use of quality indicators to assess surgical care allows comparison with standards and with other centers and monitoring changes post intervention. Objective: To apply quality indicators to breast cancer surgery and evaluate usefulness. Material and Methods: Selected indicators obtained from EUSOMA 2008 workshop were applied to 213 consecutive surgical treatment breast cancer patients from Hospital Militar de Santiago de Chile between 2006 and 2011, comparing results with previously defined standards. Results: Benign/malignant index in surgical biopsies: 1: 2.27 (minimum standard: 1/2; Optimum: 1/4), patients with complete pathologic report percentage: $99,2 \%$ (minimum: $95 \%$, optimum: $98 \%$ ), breast conserving surgery percentage: $80.20 \%$ (minimum: $70 \%$, optimum: $80 \%$ ), patients with sufficient axillary sampling percentage: $92.4 \%$ (minimum: $95 \%$, optimum: $98 \%$ ), correct axillary dissection indication percentage: $100 \%$ (minimum: $95 \%$, optimum: $98 \%$ ) and patients who underwent single surgery percentage: 90.40\% (minimum: $80 \%$, optimum: $90 \%$ ), most of them ranged between established standards. Conclusion: The use of quality indicators allows breast cancer surgery result evaluation, enabling comparison between centers and established standards, giving objective and reproducible information, helpful to plan process optimization. These or similar indicators are useful in all breast cancer treatment steps and for breast cancer unit accreditation processes. Our indicator values that are under the standard reveal that some specific local indicators are required.

Key words: Breast cancer, surgery, quality indicators.

\section{Resumen}

Introducción: Aunque existen guías clínicas ampliamente difundidas para el manejo del cáncer de mama, las variaciones entre centros impactan en los resultados. El uso de indicadores de calidad, permite

*Recibido el 10 de septiembre de 2012 y aceptado para publicación el 11 de noviembre de 2012.

Los autores no refieren conflictos de interés

Correspondencia: Dra. Ana María Donoso R. Los Guanacos 382, Peñalolén, Santiago, Chile. amdonoso@yahoo.com 
compararse con estándares, con otros centros y evaluar los cambios posteriores a una intervención. Objetivos: Aplicar indicadores de calidad al tratamiento quirúrgico del cáncer de mama evaluando su utilidad. Material y Métodos: Se aplicó indicadores de calidad a 213 pacientes consecutivos sometidos a cirugía por cáncer de mama en el Hospital Militar de Santiago entre mayo/2006 y abril/2011, comparando los resultados con estándares. Resultados: Se calculó: índice benignidad/malignidad en biopsias quirúrgicas: 1:2,27 (mínimo 1:2; óptimo 1:4), porcentaje pacientes con informe patológico completo 99,2\% (mínimo: 95\%, óptimo: 98\%), porcentaje cirugía conservadora 68,42\% (mínimo: 70\%, óptimo: 80\%), porcentaje pacientes con muestreo axilar suficiente 92,40\% (mínimo: 95\%, óptimo: 98\%), porcentaje pacientes con indicación adecuada de disección axilar 100\% y porcentaje pacientes que requirió una sola cirugía 90,40\% (mínimo: 80\%, óptimo: 90\%). La mayoría cumplió los estándares establecidos. Conclusión: La utilización de indicadores de calidad permite evaluar resultados a través del tiempo, compararse con otros centros, y con los estándares establecidos. Proporciona información objetiva y reproducible que permite evidenciar los puntos críticos en los procesos y focalizarse en ellos. El uso de indicadores de calidad puede ampliarse a todas las etapas del tratamiento del cáncer de mama y servir para unificar criterios en acreditación. El análisis de los valores que resultaron bajo el estándar reveló la necesidad de proponer nuevos indicadores útiles a nivel local.

Palabras clave: Cáncer de mama, indicadores de calidad, tratamiento.

\section{Introducción}

En cáncer de mama, aunque existen criterios estandarizados de tratamiento, basados en la evidencia y difundidos por las sociedades científicas, el 15\% de las pacientes en Estados Unidos y un porcentaje no evaluado en nuestro país no recibe el tratamiento sugerido que es el que según la evidencia, tiene los mejores resultados ${ }^{1}$.

La evaluación de la calidad de las prestaciones de salud ha cobrado importancia en los últimos años por varias razones. Por un lado, la creciente facilidad de acceso a la información ha generado una población de pacientes que conocen bien su patología y las alternativas de tratamiento; los que disponen de la posibilidad de elegir donde tratarse, requieren de información objetiva sobre el desempeño de cada centro para tomar su decisión. Por otro lado, las instituciones proveedoras de salud, han avanzado en el proceso de acreditación, estableciendo protocolos y guías clínicas cuyo cumplimiento debe objetivarse. También es sabido que el sólo evaluar la calidad, produce ya una mejoría en los indicadores, y que la adherencia a los indicadores mejora la sobrevida en una magnitud mayor incluso, a la que produce el uso de algunas nuevas drogas de quimioterapia como los Taxanos ${ }^{2}$. Finalmente, uno de los objetivos más importantes de la evaluación de la calidad, es detectar los puntos donde los procesos están fallando para focalizar allí los esfuerzos y mejorarlos.

La evaluación de la calidad en cirugía ${ }^{3}$ puede realizarse con distintos indicadores:

- De estructura: incluye todos los recursos involucrados en la prestación de los servicios, desde la planta física a los recursos humanos. Aunque tienen la ventaja de ser de fácil acceso, no existen estudios serios sobre su relación con los resultados. Además, no son fácilmente modificables, lo que los limita para ser usados en programas de mejoría de la calidad.

- De procesos: evalúa las actividades realizadas durante la atención del paciente. Describe la atención que el paciente realmente recibe. Tiene la ventaja de que permite fácilmente modificaciones de las conductas que llevan a mejorías en los indicadores. Además existe clara evidencia de su relación con los resultados finales.

- De resultados: evalúa el producto final de la atención. Históricamente se han usado en cáncer de mama, y su sola evaluación produce por si sola mejoría de los indicadores, pero no entrega información específica que permita implementar cambios.

Por estas razones y en relación a los objetivos de nuestro estudio, decidimos utilizar indicadores de procesos. Dentro de éstos, seleccionamos algunos que fueran fácilmente medibles y objetivables, que tuvieran validez basada en la evidencia, que tuvieran relación con los resultados y que se pudieran aplicar en forma uniforme.

\section{Material y Método}

Existen distintas propuestas de indicadores de calidad en Cáncer de mama ${ }^{2,4-6}$. Se seleccionó dentro de los indicadores propuestos por la Sociedad Europea de Mastología (EUSOMA), desarrollados en el contexto de la acreditación de las Unidades de Patología Mamaria Europeas, los que se relacionaran con el tratamiento quirúrgico del cáncer de mama, fuera posible recolectar fácilmente en forma retrospectiva y pudieran volver a evaluarse en forma periódica.

Se obtuvo la información de las estadísticas del pabellón, de las 371 cirugías realizadas en el período 
comprendido entre el 1 de mayo de 2006 y el 30 de abril de 2011, con los códigos correspondientes a mastectomía parcial o total con disección axilar, y los de mastectomía parcial o total sin disección axilar. De estos pacientes, en 221 , casi un $60 \%$, se logró recuperar el informe completo de las biopsias realizadas, de las cuales 164 tenían diagnóstico de cáncer de mama. En éstos, se calculó los indicadores seleccionados, con la información obtenida del informe histopatológico.

Los indicadores que se calcularon fueron:

- Indicadores relacionados con el diagnóstico:

1. Índice de Benignidad versus Malignidad en las biopsias quirúrgicas.

2. Porcentaje de pacientes con informe patológico completo.

- Indicadores relacionados con manejo quirúrgico apropiado:

3. Porcentaje de pacientes que requirió una sola cirugía.

4. Porcentaje de pacientes con muestreo axilar suficiente.

- Indicadores relacionados con evitar el sobre tratamiento

5. Porcentaje de cirugía conservadora.

6 . Porcentaje de pacientes con indicación adecuada de disección axilar.

Para el cálculo de los indicadores se obtuvo los siguientes datos:

- $\mathrm{N}^{\circ}$ de pacientes con biopsia con resultado benigno: 65 .

- $\mathrm{N}^{\mathrm{o}}$ de pacientes con biopsia con resultado maligno: 148.

- $\mathrm{N}^{\mathrm{o}}$ de pacientes con diagnóstico de carcinoma infiltrante: 133.

- $\mathrm{N}^{\mathrm{o}}$ de pacientes con diagnóstico de carcinoma in situ: 13 .

- $\mathrm{N}^{0}$ de pacientes con cáncer infiltrante que tienen consignado en el informe de la biopsia: tipo histológico, tamaño tumoral, porcentaje del componente in situ, grado histológico y estadío ganglionar. (No se incluyó los receptores hormonales ni el cerbB2, porque son realizados fuera del hospital y no están consignados en el informe de biopsia con el que trabajamos): 132.

- $\mathrm{N}^{\mathrm{o}}$ de pacientes que fueron sometidos a una sola cirugía resectiva para el tratamiento de su cáncer de mama: 132.

- $\mathrm{N}^{\mathrm{o}}$ de pacientes que fueron sometidas a disección axilar y se obtuvo al menos 10 linfonodos: 61 .

- $\mathrm{N}^{\mathrm{o}}$ de pacientes con diagnóstico de carcinoma infiltrante, con tumor único de menos de $3 \mathrm{~cm}$ : 71.
- $\mathrm{N}^{0}$ de pacientes con diagnóstico de carcinoma infiltrante, con tumor único de menos de $3 \mathrm{~cm}$ que tuvieron cirugía conservadora: 57.

- $\mathrm{N}^{\mathrm{o}}$ de pacientes con diagnóstico de carcinoma in situ, que no tuvieron disección axilar: 13.

\section{Exclusiones}

En el cálculo del indicador número 1, sobre relación benignidad versus malignidad en las biopsias quirúrgicas, se consideró entre las lesiones benignas el tumor phyllodes benigno y el carcinoma lobulillar in situ, y dentro de las lesiones malignas, el tumor phyllodes borderline y el tumor phyllodes maligno.

En el cálculo del indicador número 2 en relación al informe de biopsia, no se incluyó en los requisitos, el informe de los receptores hormonales, ya que durante ese período, algunos eran realizados en convenio con distintos laboratorios externos según la previsión del paciente y por eso no están registrados en el informe de anatomía patológica, que fue de donde obtuvimos los datos para el cálculo de los indicadores. La determinación del cerb2, aunque se solicitaba en pacientes seleccionados, sólo se realiza de rutina desde hace poco, y también se procesa en un laboratorio externo, por lo que también se excluyó de los parámetros obligatorios.

Se excluyeron del cálculo del indicador sobre el número de cirugías necesarias para completar el tratamiento quirúrgico (indicador número 3), a los pacientes que tuvieron una segunda cirugía por una recidiva local, aunque esta fuera dentro del período estudiado, si la indicación de ésta no nacía de la biopsia inicial. También se excluyeron estos pacientes del cálculo del indicador sobre la cirugía conservadora (indicador número 5), considerando que en este caso, la cirugía radical es mandatoria.

\section{Cálculo de los indicadores}

Las fórmulas utilizadas para el cálculo de los indicadores fueron las siguientes:

- $\mathrm{N}^{\mathrm{o}}$ de pacientes con biopsia quirúrgica de mama con resultado benigno/ $\mathrm{N}^{\circ}$ de pacientes con biopsia quirúrgica de mama con resultado maligno.

- $\mathrm{N}^{0}$ pacientes con diagnóstico de cáncer infiltrante e informe de biopsia completo x $100 / \mathrm{N}^{\circ}$ de pacientes operados con diagnóstico de cáncer de mama infiltrante.

- $\mathrm{N}^{0}$ de pacientes que requirieron una sola cirugía resectiva $\times 100 / \mathrm{N}^{\circ}$ total de pacientes operados por cáncer de mama.

- $\mathrm{N}^{0}$ pacientes con disección axilar en los que se examinó al menos 10 linfonodos x 100/ $\mathrm{N}^{\circ}$ total de pacientes sometidos a disección axilar.

- $\mathrm{N}^{\mathrm{o}}$ de pacientes con cáncer infiltrante no multicéntricos menores de $3 \mathrm{~cm}$ que tuvieron cirugía conservadora $\mathrm{x} 100 / \mathrm{N}^{\circ}$ total de pacientes con cáncer infiltrante operados. 
- $\mathrm{N}^{\mathrm{o}}$ de pacientes con diagnóstico de cáncer in situ a los que no se les realizó disección axilar x 100/ $\mathrm{N}^{\circ}$ total de pacientes con diagnóstico de cáncer in situ.

\section{Resultados}

Los indicadores obtenidos estuvieron en su mayoría sobre el estándar mínimo ( 5 de 6 indicadores) y algunos sobre el estándar óptimo (4 de 6 indicadores).

El análisis de cada indicador en particular, proporciona información importante sobre el funcionamiento de la Unidad de Patología Mamaria.

1. Proporción de resultados benignos versus malignos en las biopsias quirúrgicas de mama. Nos habla de la precisión diagnóstica. Lo ideal sería que una de cada cuatro biopsias quirúrgicas realizadas fuera benigna y en nuestra unidad la proporción fue de 1: 2,27. La mayoría de los casos con sospecha de neoplasia fueron a biopsia percutánea inicialmente. Los casos que aumentaron la proporción de biopsias quirúrgicas con resultado benigno, fueron las mastectomías parciales que se operaron con diagnóstico preoperatorio de fibroadenoma, las discordancias anatomo clínicas y las biopsias radioquirúrgicas. Estas últimas realizadas por imposibilidad técnica de realizar una biopsia esteroatáxica o por conveniencia económica de la paciente (nuestra población no es beneficiaria del GES y no contamos con el equipo por lo que se derivan las pacientes al área privada). Para un cálculo más preciso, las resecciones de fibroadenomas (tumorectomías) no deberían codificarse como mastectomías parciales. La indicación quirúrgica en estos casos no está dada por la sospecha de cáncer sino por otras causas.

2. Porcentaje de biopsias completas: Los datos utilizados en todos los indicadores fueron obtenidos del informe de biopsia. Éste se realiza en forma estandarizada que incluye todos los parámetros requeridos, por lo que en casi el $100 \%$ de los casos estaba completo. Los receptores hormonales y el cerb2 no se incluyeron por las razones ya expuestas.

3. Porcentaje de pacientes que requirió una sola cirugía dentro del tratamiento primario: Este indicador estuvo sobre el mínimo y cerca del estándar óptimo. Esto se relaciona con la coordinación de un equipo multidisciplinario que incluye estudio imagenológico con Resonancia Magnética en casos seleccionados, estudio preoperatorio con biopsia percutánea, marcación preoperatoria de lesiones no palpables, estudio radiológico intraoperatorio de la pieza quirúrgica y biopsia contemporánea de los bordes en todas las cirugías conservadoras.
4. Porcentaje de pacientes sometidos a disección axilar en los que se obtuvo al menos 10 linfonodos: Este indicador estuvo bajo el estándar con $92,4 \%$ siendo lo mínimo $95 \%$ y lo óptimo $98 \%$. El número de disecciones axilares realizadas es bajo porque se realiza la técnica de linfonodo centinela en todos los pacientes en los que está indicado, lo que limita la validez de este indicador. En el período estudiado, se realizó 66 disecciones axilares, de las cuales en 5 se obtuvo menos de 10 linfonodos ( 9 en 2 pacientes y 7 en 3 pacientes). Se decidió en Comité oncológico no reexplorar la axila en todos los casos. La indicación de la disección axilar estuvo dada por la presencia de linfonodo centinela positivo en 2 pacientes, y por linfonodo axilar palpable en 3 pacientes. Una de las pacientes tenía 86 años, un tumor grado 3 y 2 de 7 linfonodos comprometidos, pudiéndose inferir que la posible recidiva axilar no era un factor determinante en su pronóstico, como una manera de explicar que la disección no fuera exhaustiva para no aumentar la morbilidad de la cirugía. En el resto de las pacientes no hay factores claros que expliquen la escasa cantidad de linfonodos resecados. Probablemente ya se ha introducido en nuestro bagaje de conocimientos utilizado para la toma de decisiones, el concepto de que la disección axilar en sí, no mejora la sobrevida, y sí puede producir secuelas que afecten la calidad de sobrevida.

5. Porcentaje de cirugía conservadora en pacientes con carcinoma infiltrante no multicéntricos, menores de $3 \mathrm{~cm}$ : A pesar de que la disponibilidad de cirugía de reconstrucción mamaria inmediata y de cirugía oncoplástica produce que, en casos límite, se opte por la mastectomía, en vez de forzar una cirugía conservadora con malos resultados estéticos o con márgenes libres estrechos, este indicador está levemente sobre el valor óptimo, con $80,2 \%$. Este indicador confirma la necesidad de elaborar indicadores propios específicos para cada centro, que evalúen en forma adecuada la realidad local.

6. Porcentaje de disección axilar bien indicada: Este indicador al igual que el anterior, pretende evaluar la precisión de la magnitud de la resección quirúrgica, considerando como bien indicada la disección realizada en pacientes con diagnóstico de carcinoma infiltrante. Está sobre el estándar con un $100 \%$, lo que es esperable por el alto porcentaje de biopsias percutáneas preoperatorias. Hay que considerar que en 21 de los 66 pacientes a los que se les disecó la axila, se realizó antes la biopsia del linfonodo centinela que resultó positiva (Tabla 1). 
Tabla 1

\begin{tabular}{|c|c|c|c|}
\hline Indicador & Resultado & Estándar mínimo & Estándar óptimo \\
\hline Benigno/maligno & $1: 2,27$ & $1: 2$ & $1: 4$ \\
\hline$\%$ Biopsia completa & $99,2 \%$ & $95 \%$ & $98 \%$ \\
\hline \% Cirugía única & $90,4 \%$ & $80 \%$ & $90 \%$ \\
\hline \% Disección axilar adecuada & $92,4 \%$ & $95 \%$ & $98 \%$ \\
\hline \% Cirugía conservadora & $80,2 \%$ & $70 \%$ & $80 \%$ \\
\hline \% Disección axilar bien indicada & $100 \%$ & $95 \%$ & $98 \%$ \\
\hline
\end{tabular}

La mayoría de los indicadores presentó valores sobre el estándar mínimo ( 5 de 6 indicadores) y algunos sobre el estándar óptimo (4 de 6 indicadores). Sólo el porcentaje de disección axilar adecuada (al menos 10 linfonodos en la anatomía patológica de la disección axilar), estuvo levemente bajo el estándar.

\section{Discusión}

La idea de evaluar indicadores de calidad surge de la observación de la variabilidad de las conductas en el manejo de las pacientes con patología mamaria. Existe numerosa evidencia sobre los tratamientos recomendados, que fue revisada y adaptada a la realidad nacional en el último consenso sobre cáncer de mama el año 2009, pero la percepción es que por desconocimiento, rebeldía o falta de criterio, a veces estas recomendaciones no se siguen.

El porcentaje de pacientes en las que el tratamiento no es el estándar, es mayor en las pertenecientes al nivel socioeconómico bajo, minorías raciales o no asegurados ${ }^{7}$. Las características de la población estudiada, pertenecientes a un Hospital Institucional de las Fuerzas Armadas, nos hace pensar que en poblaciones beneficiarias del servicio de salud, estos indicadores podrían ser más bajos.

Con respecto a la naturaleza retrospectiva de este estudio, consideramos que es por un lado, una limitante, por la dificultad en la recolección de los datos; pero por otro una fortaleza, pues asegura que los datos obtenidos, representan la conducta habitual en este centro, y no existe el efecto de mejoría que se observa con sólo anunciar que serán evaluados ciertos parámetros. Para aprovechar este efecto, lo ideal es hacer evaluaciones periódicas de los indicadores más representativos y que determinen mayor impacto en los resultados finales del tratamiento, acompañado de análisis de los resultados obtenidos con todo el equipo, y planificación de las modificaciones orientadas a mejorarlos.

Privilegiamos dentro de los criterios para decidir qué indicadores evaluar, la facilidad de obtención de los datos. Son pocos los centros que cuentan con una base de datos computarizada de sus pacientes, que sería lo óptimo para este objetivo, pero los informes de las biopsias contienen gran cantidad de información que está disponible fácilmente, generalmente digitalizada y no se requiere la revisión de las fichas. Además, la información está almacenada y fácilmente se puede acceder a ella para revisar algún punto dudoso.

El análisis detallado de los resultados obtenidos en cada indicador, nos sugiere que es necesario elaborar indicadores válidos para cada centro en particular, determinados por las características específicas de cada uno, que nos permitan evaluar los aspectos críticos. Además debemos calcular algunos indicadores básicos que nos permitan compararnos con otros centros del país e internacionales.

Como un primer paso hacia proponer una evaluación global del manejo del cáncer de mama a nivel nacional, iniciamos esta autoevaluación, que a su vez permite al analizar los resultados, definir conductas conducentes a mejorar los aspectos deficientes.

Dentro de las propuestas emanadas de este trabajo, está la de confeccionar una base de datos de nuestros pacientes en forma prospectiva, que nos permita calcular fácilmente los indicadores que nos interesan, incorporando algunos tan importantes como los que evalúan el tratamiento adyuvante y el seguimiento, y tener fácilmente accesibles, en el largo plazo, la sobrevida global y la sobrevida libre de enfermedad.

También nos parece importante definir a nivel de la Sociedad de Mastología, estándares mínimos y óptimos que permitan inicialmente que cada centro se autoevalúe para en un futuro plantear la necesidad de acreditación ${ }^{8-17}$.

\section{Conclusiones}

El cálculo de indicadores de calidad en el tratamiento del cáncer de mama es factible de realizar. Deben elegirse indicadores que permitan recolección 
sencilla de los datos y reproducibilidad, y que representen los procesos que se desea evaluar.

El análisis de los indicadores calculados permite tener información objetiva y precisa sobre los procesos escogidos, evidenciando las falencias, lo que permite planificar los cambios necesarios para mejorarlas.

El uso de indicadores de calidad puede extenderse a todas las etapas del manejo del cáncer de mama, permitiendo realizar seguimiento de la evolución de los procesos a través del tiempo y comparación con otros centros a nivel nacional e internacional.

El establecimiento de estándares mínimos y óptimos de calidad, es útil en los procesos de acreditación de las unidades de patología mamaria.

$\mathrm{El}$ análisis de los indicadores obtenidos en nuestro centro nos indica que a pesar de estar en su mayoría sobre el estándar, hay varios puntos factibles de mejorar y que es necesario elaborar indicadores específicos para cada centro.

\section{Agradecimientos}

A la Sra. Lorena Ortiz, secretaria del Servicio de Anatomía Patológica, por su inapreciable ayuda en la obtención de los datos.

\section{Referencias}

1. Cheng SH, Wang CJ, Lin JL, Horng CF, Lu MC, Asch $\mathrm{SM}$, et al. Adherence to quality indicators and survival in patients with breast cancer. Med Care 2009;47:21725.

2. Curso: Calidad en los procesos asistenciales con mención en acreditación. Medwave. Módulo 2: Monitoreo de calidad.

3. De Kok M, Scholte RW, Sixma HJ, Van der Weijden T, Spijkers KF, Van de Velde CJ, et al. The patient's perspective of the quality of breast cancer care. The development of an instrument to measure quality of care through focus groups and concept mapping with breast cancer patients. Eur J Cancer 2007;43:1257-64.

4. Chen F, Mercado C, Yermilov I, Puig M, Ko CY, Kahn $\mathrm{KL}$, et al. Improving breast cancer quality of care with the use of patient navigators. Am Surg. 2010;76:1043-6.

5. Rosselli Del Turco M, Ponti A, Bick U, Biganzoli L, Cserni G, Cutuli B, et al. Quality indicators in breast cancer care. Eur J C. 2010;46: 2344-56.
6. Malin JL, Schneider EC, Epstein AM, Adams J, Emanuel E, Kahn K, et al. Results of the National Initiative for Cancer Care Quality: how can we improve the quality of cancer care in the United States? J Clin Oncol. 2006;24:626-34.

7. Edwards BK, Brown ML, Wingo PA, Howe HL, Ward E, Ries LA, et al. Annual report to the nation on the status of cancer, 1975-2002, featuring populationbased trends in cancer treatment. J Nati Cancer Inst. 2005;97:1407-27.

8. Albert JM, Das P. Quality assessment in oncology. Int J Radiat Oncol. Biol Phy. 2012;83:773-81.

9. Barni S, Venturini M, Molino A, Donadio M, Rizzoli S, Maiello E, et al. Importance of adherence to guidelines in breast cancer clinical practice. The Italian experience (AIOM). Tumori 2011;97:559-63.

10. Beckmann MW, Brucker C, Hanf V, Rauh C, Bani MR, Knob S, et al. Quality assured health care in certified breast centers and improvement of the prognosis of breast cancer patients. Onkologie 2011;34:362-7.

11. Brucker SY, Wallwiener M, Kreienberg R, Jonat W, Beckmann MW, Bamberg M, et al. Optimizing the quality of breast cancer care at certified german breast centers: a benchmarking analysis for 2003-2009 with a particular focus on the interdisciplinary specialty of radiation oncology. Strahlenther Onkol. 2011;187:89-99.

12. Chen F, Puig M, Yermilov I, Malin J, Schneider EC, Epstein AM, et al. Using breast cancer quality indicators in a vulnerable population.Cancer 2011;117:3311-21.

13. Chung KP, Lai MS, Cheng SH, Tang ST, Huang CC, Cheng AL, et al. Organization-based performance measures of cancer care quality: core measure development for breast cancer in Taiwan.Eur J Cancer Care (Engl) 2008; 17:5-18.

14. Clifford EJ, De Vol EB, Pockaj BA, Wilke LG, Boughey JC. Early results from a novel quality outcomes program: the American Society of Breast Surgeons' Mastery of Breast Surgery. Ann Surg Oncol. 2010;17 Suppl 3:233-41.

15. Crowe P. Improving surgical outcomes for patients with cancer: an Australian perspective. J Surg Oncol. 2009;99:478-80.

16. Moran M, Goss D, Haffty B, Kaufman C, Winchester D. Quality measures, standards, and accreditation for breast centers. Int J Radiat Oncol Biol Phys. 2010;76:1-4.

17. Iyengar R, Lund MJ, Lamson P, Holmes L, Rizzo M, Bumpers $\mathrm{H}$, et al. Using National Quality Forum breast cancer indicators to measure quality of care for patients in an AVON comprehensive breast center. Breast J. 2010;16:240-4. 